\title{
Philonsorbonne
}

13 | 2019

Année 2018-2019

\section{À propos du livre de Chantal Jaquet : Les transclasses}

Gérard BRAS

\section{(2) OpenEdition}

Journals

Édition électronique

URL : https://journals.openedition.org/philonsorbonne/1294

DOI : 10.4000/philonsorbonne.1294

ISSN : 2270-7336

\section{Éditeur}

Publications de la Sorbonne

\section{Édition imprimée}

Date de publication : 30 janvier 2019

Pagination : 131-145

ISSN : 1255-183X

\section{Référence électronique}

Gérard BRAS, «À propos du livre de Chantal Jaquet : Les transclasses », Philonsorbonne [En ligne], 13

2019, mis en ligne le 07 février 2019, consulté le 11 juin 2021. URL : http://journals.openedition.org/ philonsorbonne/1294; DOI : https://doi.org/10.4000/philonsorbonne.1294 


\title{
À propos du livre de Chantal Jaquet : Les transclasses
}

\author{
Gérard BRAS
}

Pas des questions, pas un commentaire : je voudrais que mon propos soit, ici, comme l'effet du livre de Chantal. Non pas moi parlant de son livre, mais le livre, par ma bouche parlée, exposant une manière de sa propre puissance, au risque de constater que son appropriation par le lecteur que je suis l'a trahi. Mais la philia que je porte à son auteure, si elle ne me garantit pas contre la trahison possible, me permet de courir ce risque devant elle.

Pour commencer, dire qu'il s'agit d'un beau livre de philosophie. Le dire, comme on dit d'un raisonnement mathématique qu'il est beau, ce qui enveloppe aussi qu'il soit vrai.

En quel sens dire qu'il est beau, qu'un livre de philosophie peut être beau, et pas seulement vrai ? Prendre beau comme les mathématiciens et comme Spinoza : pour les premiers, est beau un raisonnement qui conclut en vérité selon une économie de moyens qui assure son efficacité ; ou plutôt, est beau un raisonnement qui, avec un appareillage conceptuel léger, permet de conclure un énoncé riche en conséquences; pour le second, est beau ce qui convient avec la complexion du spectateur : il n'est pas de beau en soi, mais relativement à un individu qui l'éprouve tel, à raison de la joie que la chose dite belle lui procure. Alors, disons que je trouve le livre de Chantal beau parce qu'il convient avec ma complexion, mon ingenium, parce qu'il me procure une joie intellectuelle due à la fois à la meilleure compréhension d'un phénomène social objectif et aussi à la meilleure compréhension de moi-même, moyen, entre autres, pour essayer de devenir sage, c'est-à-dire «conscient et de soi, et de Dieu, et des choses avec certaine nécessité 
éternelle $»^{1}$. Je le trouve beau, comme livre de philosophie, parce que je le lis comme livre d'une spinoziste qui use de son savoir et de sa culture pour éclairer une question qui me touche. Et parce qu'elle le fait sur un mode non académique, en interrogeant précisément le statut de ceux qui, dans la faculté, ont emprunté des routes de côté. De le faire en philosophe, de donner au public un livre de philosophie authentique, non un livre de sociologie ou une œuvre de littérature. Et je ne crois pas, ou ne crains pas de tomber, ceci disant, sous le coup de l'accusation de Bourdieu à l'encontre de ces philosophes qui se croient autorisés à mépriser les «sciences sociales », pour cette raison que je crois, en accord avec Chantal, que la sociologie est bien l'une des entrées possibles dans la question que le livre pose, tout comme la littérature, qu'il n'est pas d'autre voie pour la vérité de la question que la multiplicité des entrées qui refuse l'exclusivité d'une discipline. Encore qu'il faille remarquer que la sociologie, contrairement à la littérature, se penche plus sur la reproduction que sur ce qui semble lui faire exception.

Ni sociologie, ni psychologie, ni littérature, mais philosophie. Comment caractériser positivement cette démarche ? Si les sciences sociales décrivent un phénomène qu'elles ont préalablement circonscrit, en exposant les causes cachées, les rapports sociaux qui en rendent raison, si la littérature montre les effets, notamment subjectifs, de ces rapports, la philosophie interroge les conditions qui rendent cette connaissance possible, s'efforce d'élaborer les questions et les concepts qui permettent de mener l'enquête. C'est donc par un va-et-vient permanent entre science, littérature et philosophie que la pensée du livre s'élabore, la philosophie se nourrissant de ce qui n'est pas elle, comme le disait Georges Canguilhem. Donc pas de concurrence pour le monopole entre ces trois disciplines, concurrence académiquement instituée et désastreuse pour la pensée.

Je m'efforcerai ainsi de lire le livre de Chantal comme un livre de philosophie; non pas que cette lecture soit meilleure que l'autre, sociologique, mais simplement parce que c'est celle que je peux pratiquer le moins mal. Ce faisant, je suivrai la discrète invitation qu'il m'adresse à moi lecteur : le lire en philosophant à la première personne, en philosophant en prenant en considération les affects qu'il suscite et qu'il enveloppe. Non, je l'espère, pour faire étalage égotique, ce qui ne serait guère philosophique, mais pour l'entendre comme effort pour penser un universel concret, incarné ; ou pour le dire avec un mot commun à Canguilhem et Althusser, l'entendre comme un livre «de parti pris », ce qui n'est sans doute pas la meilleure philosophie, mais qui est certainement « la vraie philosophie $»^{2}$. Je voudrais m'efforcer de le comprendre en partant de la dédicace : À Renée Thomas ma tante, qui franchit le Bonrieu pour la première fois, à tous les transclasses, parce que ma mère, couturière, fille cadette d'une famille

1. Spinoza, Éthique, $\mathrm{V}^{\mathrm{e}}$ partie, scolie de la proposition 42.

2. Spinoza : «Je ne prétends pas avoir trouvé la philosophie la meilleure, mais je sais que j'ai connaissance de la vraie », Lettre 76 à Albert Burgh. 
de paysans savoyards, a franchi le Rieu pour s'embaucher à la ville comme bonne, avant de pouvoir y exercer son métier et d'épouser cet ouvrier métallurgiste, mon père, venu à la ferme en 1943, pour échapper au S.T.O.

Cette première personne qui s'absente de l'écriture manifeste du livre, présentée juste dans l'interstice qui sépare la page de garde de l'introduction, invite à faire de même : raisonner, c'est-à-dire élaborer selon l'ordre et la connexion de la raison les concepts qui permettent de comprendre non seulement le général, mais aussi le singulier. C'est à cette invitation que je voudrais répondre en essayant d'ajouter deux ou trois pierres à l'édifice que Chantal Jaquet a construit, et que je considérerais volontiers comme work in progress ${ }^{3}$.

\section{Les conditions de possibilité des transclasses}

Le livre de Chantal Jaquet se divise en deux parties, l'une portant sur les causes qui permettent d'expliquer qu'il y a des transclasses, l'autre sur la complexion, l'ingenium qui détermine le transclasse à être ce qu'il est. De l'existence à l'essence en quelque sorte. En quelque sorte, mais pas vraiment: ce modèle métaphysique ne convient pas au fond du raisonnement, pas plus que ne conviendrait un modèle phénoménologique. En effet, il ne s'agit pas de trouver une nature au terme d'un parcours qui, débutant avec l'apparence en dégage la raison, ni d'interroger l'apparaître pour orienter la pensée vers son être, mais de déterminer les causes qui font qu'une espèce de choses finies, désignées de façon neutre par le néologisme «transclasses », existe et existe avec tels caractères et non d'autres, étant entendu que ce n'est pas parce qu'elles ont ces caractères-là que ces choses existent, mais parce qu'elles sont déterminées à exister par ces causes-ci qu'elles se donnent dans l'existence sur ce mode-là. Il n'est donc question que de l'essence des transclasses, connue à partir de l'essence singulière de ces transclasses qui s'exposent chez Stendhal, Michelet, Annie Ernaux, Didier Eribon, Pierre Bourdieu, Paul Nizan et tous les autres cités dans le livre, auxquels j'ajouterai volontiers Martine Sonnet avec son magnifique Atelier 62 (mais la liste est ouverte, évidemment). Il n'est donc question que de l'essence, mais en un sens spinoziste, conçue comme "ce sans quoi la chose, et inversement ce qui sans la chose, ne peut ni être ni se concevoir ${ }^{4}$.

Je m'arrêterai sur un seul aspect : distinguant plusieurs causes, Chantal Jaquet met au jour deux modèles susceptibles de générer identification et imitation de la part de ceux des enfants des classes populaires qui seront déterminés à ce transport ou transfert de classe, le modèle familial et le modèle scolaire. Un peu plus loin elle évoque les conditions socio-

3. Le livre collectif que nous codirigeons, reprenant les actes du colloque organisé à Paris I les 26 et 27 juin 2017, La Fabrique des transclasses, PUF, septembre 2018, atteste de la fécondité évolutive de son travail.

4. Spinoza, Éthique, $\mathrm{II}^{\mathrm{e}}$ partie, définition 2. 
économiques sans lesquels l'imitation resterait fantasme éprouvé sur le mode du manque ou de l'échec. Parmi ces causes, le système étatique des bourses (incluant les Écoles normales, les I.P.E.S. et les Écoles normales supérieures) et les activités sociales des Comités d'Entreprise. Elle conclut ce paragraphe : «il s'agit de déterminer de manière plus générale les raisons pour lesquelles un individu s'oriente vers un modèle autre que celui qui est en vigueur dans son milieu. La présence d'un mode de vie alternatif n'est pas suffisante pour déclencher un mécanisme de non-reproduction. (...) Il faut comprendre le jeu des forces à l'œuvre dans l'adhésion à un modèle (...) Une telle démarche implique d'analyser ce qui le rend désirable en acte, en mettant au jour le nœud des déterminations qui se contrarient et se composent pour produire une puissance d'agir capable de l'accomplir $»^{5}$.

C'est la raison pour laquelle je pense qu'il faudrait ajouter au moins un modèle, que $\mathrm{j}$ 'appellerai sans doute «modèle social »; ou plutôt qu'il faut élaborer le concept englobant une pluralité de modèles, capable de rendre raison à la fois de la reproduction et de la non-reproduction marginale dont nous parlons aujourd'hui. J'irai le chercher du côté de ce que l'on appelle «société civile », en la distinguant, avec Hegel sûrement, mais aussi avec Spinoza et d'autres, de l'État ou de la Souveraineté.

Je commence par un élément juste évoqué rapidement par Chantal, mais sous la seule forme des conditions économiques : le Comité d'Entreprise. Je pense que l'on doit l'élargir dans deux directions : le C.E. est une institution qui résulte de la loi du 16 mai 1946 et qui est gérée par les syndicats. Il s'agit donc d'une instance non pas étatique, mais de la société civile qui dénote une certaine autonomie des salariés, singulièrement de ce qui se nommait à l'époque «classe ouvrière ». L'apport économique des C.E. est incontestable, mais il ne faut pas passer sous silence ce qui ressortit à leur apport symbolique : exposer la capacité des ouvriers à prendre en charge des questions qui relèvent soit de l'économie, soit, surtout, des conditions de travail et de santé au travail. Martine Sonnet en donne de belles illustrations dans son livre. C'est à la fois une instance de gestion de certaines questions et un lieu où le conflit social se déploie. Je pense donc que l'on doit tenir comme un ensemble institutionnel C.E. et syndicats, condition pour comprendre qu'ils constituent alors effectivement un modèle mimétique possible: celui de ce que, avec Gramsci, nous pouvons nommer « intellectuel organique », figure qui rend désirable, pour ces fils d'ouvriers, le devenir intellectuel, parce que leurs parents ou leurs semblables sont devenus des intellectuels, des ouvriers-intellectuels, changeant de classe sans en changer. Il est certainement de bon ton aujourd'hui de regarder toute cette histoire de haut et d'assimiler militants syndicaux et politiques ouvriers à des bureaucrates staliniens dominants et trahissant les vrais ouvriers. Lecture politique de cette histoire qui n'est pas sans fondement et que l'on peut soumettre à la discussion rationnelle. Mais, sous l'angle qui nous intéresse ici, il s'agit de tout autre chose : de la constitution d'une figure symbolique,

5. Chantal Jaquet, Les Transclasses ou la non-reproduction, PUF, 2014, p. 63. 
symbolico-charnelle pour parler comme Desanti, qui a motivé, plus ou moins consciemment, certains de ces fils d'ouvriers qui ont échappé à la reproduction sociale à laquelle l'école est orientée sur un mode majeur. Je me souviens de ma gêne lorsque, élève de lycée, il fallait remplir la fiche demandée par chaque professeur, où l'on nous interrogeait sur la profession des parents. Que dire ? Ouvrier? J'en étais fier. Permanent syndical ? Je l'estimais dangereux donc inavouable. Devenu professeur, j'ai vite cessé de poser cette question à mes élèves.

Le travail de préparation de ce texte m'a conduit à comprendre d'une autre manière ce qu'Althusser voulait dire quand, dans son article sur les Appareils idéologiques d'État, il mentionne les syndicats et les partis politiques. On se souvient que les A.I.E. interpellent l'individu en sujet, sur le mode théâtral de cette injonction, «Hep, toi, là-bas! » et, de ce fait, l'intègre au dispositif de domination, soit comme rouage dominant, soit sur le mode de la sujétion admise. Lecture sommaire sans doute générée par l'article lui-même. Lecture qui manque la contradiction que nous pouvons apercevoir ici : celle qui ouvre la voie à une forme de passage social. Cette non-reproduction à la marge ne peut supprimer l'exploitation capitaliste, ne peut transformer en ses fondements la société. Elle peut même servir d'alibi à sa persévérance en l'état. Mais elle constitue une cause, pour comprendre un transclasse qui n'est pas animé par la honte d'avoir trahi les siens, parce qu'il a vécu son passage sur le mode de la chance de pouvoir accéder à un monde de savoir et de culture universel duquel les conditions sociales et économiques qui étaient les siennes l'excluaient, voire sur le mode de la mission en vue de former des cadres pour la «Révolution », à la manière d'un Michelet réfléchissant son écriture de l'histoire comme histoire du peuple, révélant que le peuple est sujet de l'histoire et rendant possible la réappropriation de l'histoire par le peuple.

Ce qui suppose aussi, puisque j'ai cité Desanti, qu'il soit soutenu dans son effort par une croyance, voire une double croyance : croyance en les vertus du savoir, en son caractère universel, rationnel, transclasse, à l'opposé de celle, un moment professée par le parti communiste d'une science de classe; croyance aussi en une téléologie historique, commune à la République et aux hommes de gauche qui se définissaient comme révolutionnaires, qui présentait un horizon d'attente ou d'espérance susceptible de les soutenir dans leur effort. Du coup ces transclasses se réfléchissaient moins comme traîtres à leur classe, figure incarnée par l'ouvrier devenu contremaître, ou le nouveau riche détenteur d'actions cotées en bourse, mais comme étant situés aux avant-postes de l'histoire.

Si je synthétise ces éléments, je crois en effet que les causes de l'existence des transclasses se trouvent du côté d'une société civile qui se donne, avec le soutien ou contre l'État selon les cas, des institutions autonomes qui forment des modes de transition entre l'individu et l'État, institutions qui contribuent fortement à former ce que Bourdieu appelait le «capital symbolique » en même temps que le réseau capillaire qui intègre les individus à une société. Si c'est vrai, je crois que l'on ne peut se 
contenter de distribuer, comme parfois Chantal le fait, les individus en groupes dominant et dominé. La finesse du concept de classe, tel que Chantal en fait usage, et qui demanderait peut-être précision dans sa définition, conduit à poser des classes intermédiaires, bien sûr intégrées au dispositif de domination, mais qui ne permettent pas de penser simplement le fait de sortir d'une classe dominée ou exploitée, comme passage dans le camp des dominants. Il faudrait sans doute reprendre le terme utilisé par Bourdieu de « dominés chez les dominants».

Le dispositif que j'ai évoqué du côté de la société civile rend compte, je crois, de la manière avec laquelle un individu peut être subjectivé dans son effort pour changer de classe. Il va sans dire qu'un autre rouage a été, et est encore essentiel : ce sont les Églises, singulièrement l'Église catholique (et il faudrait enquêter sur les institutions islamiques aujourd'hui). Les nombreuses références faites aux livres d'Annie Ernaux sont là pour l'illustrer. Je voudrais évoquer un autre cas qui me tient à cœur : celui de ces jeunes bourgeois ou petits-bourgeois qui sont devenus prêtres-ouvriers parce qu'ils ont été « appelés » par cette vérité qui, à travers la pauvreté du Christ, les a conduits à témoigner de leur foi en travaillant en usine, sans intention missionnaire, contrairement aux «établis » d'après 68. Mais je n'ai pas le temps d'en dire plus.

Je voudrais seulement souligner, pour conclure cette partie de mon propos, que l'enquête sur les transclasses fait apparaître l'importance des institutions sociales ou civiles (famille, écoles, églises, syndicats, partis, communes etc.) qui n'ont pas une existence indépendante ou séparée de l'État, que l'État intègre, mais qui s'en distingue si on considère celui-ci comme l'instance qui détient le pouvoir de légiférer en général et celles-ci comme instances organisant l'existence et les relations effectives entre individus et groupes sociaux. Dans une société qui n'est pas une société d'ordres, mais une société d'individus, le changement de classe est possible de droit et rendu possible effectivement, ou limité par le mode opératoire de ces instances où s'incarnent des modèles qui, globalement, orientent vers la reproduction. Le fait que marginalement (d'un point de vue statistique) des individus soient déterminés à désirer changer de classe, et y parviennent, met en lumière le rôle de ces instances socialisantes qui constituent comme un terreau commun, plus ou moins élargi, au sein d'une société. C'est ainsi que lorsque l'on diagnostique aujourd'hui une «panne de l'ascenseur social » on peut entendre deux choses fort différentes. La première, induite par la métaphore de l'ascenseur, dénote le fait que la structure sociale reste stable et que ceux d'en-bas restent en bas, voire restent bas. La seconde, que les institutions sociales ne jouent pas ou plus leur rôle transitionnel entre le particulier et le général, que la constitution du commun qui devrait s'opérerlà ne s'y réalise pas. Donc que chacun tend à être enfermé dans sa particularité, faute d'une croyance commune. Mais c'est en même temps souligner que cette sclérose entre ou peut entrer en contradiction avec les aspirations de nombre d'acteurs sociaux susceptibles de mettre en crise ce qui apparaît comme fatalisme sociologique. 


\section{Complexion et désir mimétique : la question de l'estime et de l'estime de soi}

De là le deuxième volet du livre qui passe de la question de la présentation d'un objet désirable, ou plutôt d'un objet pour le désir mimétique, à celle de l'expression de ce désir et de ses avatars. Il ne suffit pas en effet qu'un «modèle mimétique » soit présent, ni que les moyens de l'imitation soient disponibles, encore faut-il qu'il soit effectivement désiré pour que l'individu s'efforce de le réaliser.

Chez Bourdieu, le concept d'habitus rend raison à la fois des conduites des agents sociaux déterminés par les règles du champ dans lequel ils sont inscrits, règles incorporées, donc naturalisées et de ce qui leur apparaît comme étant désirable parmi les objets qui se présentent à eux. $L a$ Distinction a montré que ce qui est objet désirable pour les uns est méprisable ou insignifiant pour les autres. Dans les Méditations pascaliennes, Bourdieu réaffirme que l'habitus n'est pas un concept qui fonde un fatalisme sociologique (cf., entre autres, p. 186-187). Il n'en reste pas moins que l'on ne comprend pas assez clairement avec lui pourquoi, c'est-à-dire quelles causes déterminent un individu à désirer un objet qui n'est pas homogène au champ social qui est le sien, quelles causes le déterminent à former un désir hérétique, selon le mot qu'il emploie. Chantal Jaquet montre que le concept de l'habitus doit être englobé dans celui, spinoziste de complexion ou ingenium.

L'ingenium ou la complexion désignent la chaîne de déterminations qui se nouent pour former la trame d'une vie singulière. Ils gardent de la notion de génie l'idée d'originalité, mais la dépouillent de toute dimension innée et transcendante pour mettre l'accent sur la production historique d'un tissage industrieux en relation avec un milieu. Ils invitent ainsi à penser le transclasse comme un être pris dans un nœud de relations et d'affects qui se combinent et se composent pour produire une nouvelle configuration ${ }^{6}$.

Ainsi, complexion ou ingenium apparait comme un concept plus large et plus fin : plus large en ce qu'il englobe l'habitus comme un cas particulier, ou plutôt comme l'un de ses effets possibles, comme son effet majeur, celui de l'incorporation «réussie » des règles du milieu, celui de la normalité, alors que le concept de la complexion enveloppe celui de la normativité du vivant, pour reprendre une distinction essentielle chez G. Canguilhem ; plus fin en ce qu'il engage le problème de l'individualité, de la singularité individuelle, interrogeant les causes de la différence singulière en maintenant que l'individualité procède du transindividuel, et non d'une subjectivité monadique, ou étant en soi et se réalisant en sortant de soi afin de se réfléchir: elle procède tout entière des relations nouées avec le monde extérieur, avec les autres. Je ne reviens donc pas sur ce qui est dit dans le

6. Chantal Jaquet, Les Transclasses, op. cit., p. 102. 
livre concernant l'importance de ce concept de complexion qui permet d'expliquer ce qu'est la seconde nature, en faisant l'économie de l'axiome, pascalien, qui la pose comme perte de la première nature. Autrement dit, le concept d'ingenium ne fait pas que constater qu'il y a incorporation des règles par les agents sociaux, mais soutient que cette incorporation est indexée à leur désir, à leurs joies et tristesses, exprimant quelque chose comme un calcul vital automatique du moindre mal et/ou du plus désirable. Dit autrement, il pose le problème des modes de la subjectivation, faisant de l'individu singulier non pas un étant en soi donné naturellement dans sa singularité, mais le résultat d'un processus où son aptitude à être affecté et à affecter, à entrer en relation avec les choses et les autres détermine ce qu'il deviendra. Il est clair de ce point de vue que les transclasses ne sont pas de nature différente des autres individus, même s'ils peuvent être déterminés à croire que leur « réussite », assimilée à leur «ascension» sociale, est due à leur génie personnel. Et ils sont d'autant plus déterminés à le croire qu'ils considèrent le caractère exceptionnel de leur trajectoire, se considèrent comme exception. Il est clair aussi qu'en permettant de souligner les failles de la reproduction sociale, ils obligent à enquêter sur les causes de la subjectivation, donc aussi de la singularisation individuelle, causes qui valent pour tous les individus.

Or il me semble que si l'on peut constater que les individus diffèrent les uns des autres quant à leur complexion, c'est-à-dire s'ils sont déterminés par des causes différentes à devenir ce qu'ils sont devenus, ce qui veut dire que les désirs des uns sont différents des désirs des autres, on ne peut en conclure que les affects qui déterminent la subjectivation sont d'importance équivalente.

La non-reproduction invite à penser des histoires imbriquées et des causes intriquées les unes dans les autres. Elle n'est pas le produit d'une cause première et déterminante que l'on pourrait présenter comme la cause, mais d'un agencement singulier de causes multiples dont la résultante est une trajectoire. À cet égard, aucune cause, à elle seule, n'est décisive. Ni l'ambition ni la présence de modèles alternatifs familiaux ou scolaires ni l'existence d'incitations financières et de mesures socioéconomiques favorables ne permettent à elles seules de l'expliquer?

Dire qu' «il n'y a pas d'affect privilégié (...) qui puisse être brandi comme cause primordiale $»^{8}$ est, je pense, certain. C'est, à mon sens, la raison pour laquelle la problématique pascalienne, fort utile quand il s'agit de donner une description anthropologique de l'homme de la seconde nature et de ses illusions, échoue à expliquer les causes d'un désir de transclasse, et plus généralement du désir qui meut le processus de subjectivation, parce qu'elle fait de l'orgueil ou de l'amour-propre la cause première de toutes les

7. Chantal Jaquet, op. cit., p. 96-97.

8. Ibid. 
conduites humaines. Une chose est de percevoir sous les conduites qui se croient libres, le ressort de l'amour-propre «qui triomphe dans sa propre défaite » (La Rochefoucauld), une autre d'expliquer l'amour-propre, c'est-àdire d'en rechercher les causes à la manière de Spinoza.

Par conséquent, je ne crois pas que l'on puisse suivre Pascal jusqu'au terme de sa critique du moi, telle qu'elle est donnée dans le fragment 323 (B) 688 (L). Si l'on peut soutenir, avec lui, l'inanité d'un moi substantiel, peuton conclure que l'«on n'aime jamais personne que pour des qualités empruntées »? Que le moi n'est fait que d'emprunts ? Qu'il est tout entier illusion? Ce qui est bien le chemin dans lequel Pascal nous conduit : nous ne pouvons nous aimer nous-mêmes, parce qu'entre nous et notre désir se loge un écart infini, celui qui est né du désir d'être à nous-mêmes notre propre centre et de l'impossibilité de trouver en nous un tel principe; nous nous aimons donc à travers le regard des autres, nous soumettant de fait à ce que nous imaginons être aimable pour eux : «C'est la plus belle place du monde, rien ne le peut détourner de ce désir, et c'est la qualité la plus ineffaçable du cœur de l'homme » $(470 \mathrm{~L})$. Or Spinoza nous aide à comprendre que la vanité de la quête de reconnaissance, clairement décrite dans l'anthropologie pascalienne de la seconde nature, n'enveloppe pas la conséquence qu'il en tire quant au caractère illusoire de toute émancipation hors de l'amour du Christ. Ou, si l'on veut tenir séparée son anthropologie de son soubassement théologique, celle qui conclurait au caractère illusoire de toute émancipation, parce que toute entreprise humaine du genre ne consiste qu'en une quête d'estime, de gloire dans laquelle «notre propre intérêt est (...) un merveilleux instrument pour nous crever les yeux » (44 L). Si nous suivons Pascal dans la critique des illusions du moi, peut-on conclure avec lui qu'une émancipation dans l'immanence, une émancipation par et dans l'effort pour augmenter la puissance de pensée de la raison est la dernière des illusions par laquelle l'athéisme imagine exhiber sa force prétendue ?

Ce détour pour reprendre une question seulement suggérée plus haut: n'y a-t-il pas un affect qui soit dans le procès de subjectivation des transclasses, comme de tout individu, non pas cause première, mais qui joue le rôle de clé de voûte de la complexion, du réseau d'affects qui constitue l'individualité singulière? Ne peut-on trouver dans la quête de reconnaissance, ou plutôt d'estime et d'estime de soi, le principe de subjectivation qui meut l'individu dans les relations qui le constituent?

Il faut en effet remarquer dans les lectures de textes littéraires que Chantal Jaquet propose, l'importance de la honte pour les auteurs convoqués, honte prise en un sens spinoziste : non pas honte de ce que l'on est, mais de ce que l'on imagine que les autres imaginent que l'on est : « la honte est une tristesse qu'accompagne l'idée d'une action dont nous imaginons que d'autres la blâment ${ }^{9}$. » Sans doute faut-il compléter la définition de Spinoza en disant : non seulement d'une action, mais d'un état,

9. Éthique, $\mathrm{III}^{\mathrm{e}}$ partie, définition 31 des affects. 
d'être tel, d'être celui-ci alors que l'on imagine devoir être celui-là, état qui s'expose dans le parler, le maintien du corps, la tenue vestimentaire, les pratiques culturelles, tout ce qui relève de la distinction, au double sens du terme expliqué par Bourdieu. Ce que soutient Chantal Jaquet: «Le transclasse rougit de ses origines, car il imagine à tort ou à raison que les autres les jugent inférieures et qu'il a intériorisé ce jugement ${ }^{10}{ } »$. Cette honte d'état relèverait de ce que Spinoza nomme bassesse, qui consiste à « faire de soi, par tristesse, moins d'état qu'il n'est juste », affect dont il note la rareté, « car la nature humaine, en soi considérée, déploie contre [lui] tous les efforts qu'elle peut; et ainsi, ceux qu'on croit bas et humbles au plus haut degré sont en général ambitieux et envieux au plus haut degré ${ }^{11}$. Étant déterminés à fuir les tristesses et rechercher les joies, nous tendons à fuir la honte comme la bassesse ; l'expérience du transclasse pourrait être celle d'une honte et bassesse qu'on ne peut fuir puisqu'elle est honte de ce que l'on est et honte de ce que l'on a fui, de ce que l'on est devenu; en ce sens elle serait celle de l'échec nécessaire de toute tentative de fuite parce que la fuite cherche à ne pas « rester soi », pour reprendre le mot de Michelet, que nous corrigerions en ne pas être soi. Mais si nous en restions là nous serions reconduits aux limites qui sont celles de Pascal, la bassesse pouvant n'être, comme nous venons de le voir, que l'autre face de l'orgueil. C'est alors un autre affect qui aimante l'individu se singularisant : l'acquiescientia in se ipso, satisfaction de soi-même (Pautrat) ou assurance en soi-même (Macherey), que l'on pourrait aussi nommer estime de soi.

Par ce mot d'estime, j'aborde ce qui constitue sans doute une différence dans notre approche de la question des transclasses et, puisque le transclasse ne se distingue pas essentiellement de l'individu singulier, de l'individuation. Ne peut-on soutenir que l'effort pour s'estimer soi-même, inséparable sans doute de l'effort pour être estimé, est le moteur subjectif dans ce procès d'individuation, de subjectivation, de singularisation? Nous sommes bien d'accord sur un point : il ne s'agit pas, comme c'est le cas avec l'amour-propre chez La Rochefoucauld ou l'orgueil chez Pascal, d'un affect primordial qui jouerait comme cause première de ce processus. C'est bien clair puisque nous sommes déjà en présence d'un complexe d'affects : orgueil, assurance en soi-même, mais aussi non encore nommés gloire et reconnaissance et, last but not least, ambition, termes pris ici au sens spinoziste, c'est-à-dire dégagé de leur connotation axiologique morale. Je trouve donc que la question posée par ce qu'on nomme «luttes pour la reconnaissance », évoquées aux p. 108 et 109, est un peu trop rapidement évacuée. Si les luttes pour la reconnaissance sont ce que Chantal en dit, c'est-à-dire des demandes de reconnaissance des particularités (sociales, sexuelles, raciales, culturelles, religieuses etc.), alors, bien sûr, elles ne peuvent rendre compte d'aucun des phénomènes de passage de frontières

10. Chantal Jaquet, op. cit., p. 168. Voir aussi Annie Ernaux, La Honte, Gallimard, folio, p. 139-140.

11. Éthique, $\mathrm{III}^{\mathrm{e}}$ partie, définition 29 et explication, des affects. 
au sein d'une société qu'elles contribuent, au contraire, à fixer en ordres sans mettre en cause la structure de domination qui les rend possibles. Si, à la suite de Hegel, ou d'un Hegel caricaturé, elles supposent un soi pensé en son essence qu'il réalise en s'affrontant à son autre, selon un processus téléologique, alors, en effet, ce concept ne permettra pas de penser la singularité qui apparaît dans l'enquête sur les transclasses. Mais justement il est légitime de se demander si l'appropriation de Spinoza pour comprendre la complexion des transclasses ne permet pas de repenser, sur un mode non hégélien, les luttes pour la reconnaissance, ou plutôt, pour éviter certaines confusions, les luttes pour l'estime et l'estime de soi $^{12}$ ?

Poser cette question en engage une autre: celle de l'importance à accorder au concept de l'imitation des affects, qui est sans doute la clé de voûte de la complexion, telle que je la comprends. La première partie du livre évoque les modèles et de mimétisme, sans utiliser cet instrument conceptuel à mes yeux essentiel du spinozisme qu'est l'imitation des affects, au moment d'expliquer et de comprendre la complexion des transclasses. Il est vrai que l'imitation des affects n'est pas un affect: en ce sens, je le concède volontiers, ma «clé de voûte » n'est pas un affect, mais, je crois, un ressort (ou un moteur) parce qu'il s'agit d'un mécanisme qui démultiplie les relations affectives, donc qui les complique aussi. Mécanisme essentiel pour rendre raison des processus d'anthropogenèse qui supposent l'identification au semblable.

Quel bénéfice peut-on escompter de l'usage de cet instrument conceptuel, en regard de la question qui nous occupe ? J'en vois au moins deux. D'une part, il permet d'expliquer pourquoi la quête de l'estime est solidaire de celle de l'estime de soi, là où le dispositif pascalien faisait de l'orgueil un principe mystérieux sans lequel l'homme serait un mystère encore plus profond, l'assimilant par-là même au péché originel. D'autre part, En comprenant comment un individu est engagé dans un processus psychique qui le conduit de la gloire à l'ambition, il permet de réfléchir sur ce dont l'ambition est porteuse et de montrer la voie d'une possible transformation de l'ambition en fermeté et générosité, de montrer comment l'ambition peut engager sur la voie d'une reconnaissance de la raison (Lazzeri).

Par exemple, aux pages 166 et suivantes, Chantal Jaquet montre que « la complexion d'un transclasse se noue et se dénoue autour de la honte sociale », ce qui fait bien de cet affect la clé de voûte ou le point nodal de la complexion du transclasse. C'est dans l'analyse qui commence là, qui cherche explication de cette «honte sociale » que j'attendrai le passage par le mimétisme affectif. N'est-ce pas lui qui peut rendre raison des différents avatars de la honte, allant de la bassesse à l'ambition de domination en passant par l'ambition de gloire ? Il me semble que si la «honte sociale»

12. Voir Christian Lazzeri : Pascal et Spinoza, deux modèles de reconnaissance, in L. Bove, G. Bras et E. Méchoulan, Pascal et Spinoza, Pensées du contraste : de la géométrie du hasard à la nécessité de la liberté, Paris, Éditions Amsterdam, 2007. 
joue bien le rôle évoqué, c'est parce que le transclasse est en demande ou en manque d'estime, manque qui le conduit à se mésestimer, qu'il éprouve donc cette honte sociale. Et c'est parce qu'il est déterminé, comme tout un chacun à sortir de cette honte, sur le mode de la fuite ou de l'assomption de son être, qu'il cherche l'estime ou la reconnaissance. Mais comment expliquer la honte, tout comme la fierté (gloria) ? Comment comprendre que je sois affecté par les affects que j'imagine être chez ceux que j'imagine me juger ? Je ne suis pas simplement honteux devant autrui, je suis honteux parce que j'éprouve le sentiment que j'imagine être chez autrui, en même temps que j'imagine en être la cause. De la même façon, je suis glorifié (ou fier) parce que j'éprouve la joie que j'imagine être chez autrui et dont j'imagine être la cause. La honte, comme la fierté, ne sont pas réflexion mais contamination affective imaginaire. Je vais donc fuir la honte et poursuivre la gloire, avoir tendance à me glorifier au-delà de ce qui est réel et me rendre par-là pénible aux autres, leur apparaître comme prétentieux, comme celui qui ne sait pas rester à sa place. Annie Ernaux le résume : «Obsession : qu'est-ce qu'on va penser de nous? »(La Place, p. 61).

Comme le commente Macherey: «ces sentiments, en même temps qu'autrui, nous concernent aussi personnellement, et c'est précisément cette ambiguïté qui les caractérise : ils peuvent donc être rattachés, en même temps qu'à la considération d'une cause extérieure, à celle d'une cause intérieure, du fait que par mimétisme affectif, nous ressentons pour nousmêmes les affects que nous imputons imaginairement à autrui : alors c'est aussi nous-mêmes que nous aimons ou haïssons ${ }^{13} »$. Et si nous pouvons escamoter autrui, nous passons de la fierté à l'assurance en soi-même et de la honte au repentir. Le prix à payer dans cette quête passive d'une issue à la honte est celui de la soumission à autrui par le biais de la fierté, de la glorification : si nous sommes déterminés à rechercher ce qui nous procure joie, nous allons chercher à nous glorifier en cherchant à être cause de la joie chez autrui, ce qui implique que nous devrons user de stratégie pour lui complaire. Tel est sans doute le syndrome du bon élève. Si ceux à qui nous cherchons de complaire nous tirent dans des directions différentes voire contraires, nous tenterons le grand écart, ou le clivage en soi-même ${ }^{14}$ : être bonne élève et se lier d'amitié avec les plus turbulentes. On sait que l'ambition de gloire se mue en ambition de domination, dès qu'un homme, comprenant la sujétion qu'elle implique cherche non plus à complaire aux autres, mais à faire en sorte que les autres aiment ce qu'il aime lui-même. Les transclasses sont-ils «vaccinés » contre cette ambition de domination, parce qu'ils en ont été victimes ? Le croire serait oublier la complexité de l'âme humaine, et le rôle fondamental des rencontres et des circonstances dans la formation de l'ingenium. Rien n'interdit de concevoir que la domination, que l'exercice du pouvoir apparaisse à tel ou tel comme issue

13. P. Macherey, Introduction à l'Éthique de Spinoza, la troisième partie, la vie affective, Paris, PUF, p. 242.

14. Voir Annie Ernaux, La Honte, op. cit., p. 94-95, 115-116. 
imaginaire devant ce que la quête de gloire impose de sujétion. L'ambition d'un Rastignac peut en témoigner en littérature. Le mépris que certains enseignants transclasses affichent, volontairement ou non, à l'endroit de certains élèves issus d'une classe sociale similaire à celle qu'ils ont quittée, en est un autre exemple.

Il me semble donc que pour rendre compte du désir d'un individu singulier, déterminé à s'efforcer dans une orientation qui le conduit hors de sa classe d'origine, pour rendre raison de sa complexion, il faut faire une place primordiale à l'imitation affective qui peut expliquer à la fois la honte sociale et les luttes pour l'estime et l'estime de soi dans lesquelles elle s'exprime. Outre les conditions objectives qui rendent possibles le passage, exceptionnel, d'une classe dans une autre, il faut que les rencontres engagent le désir mimétique hors des limites du milieu social et familial d'origine, lequel apparaîtra alors, et alors seulement, comme affecté d'un manque. La manière avec laquelle ce manque est imaginé conditionne l'écart intérieur qu'il entretiendra avec ses origines, allant du rejet, à la façon d'un Julien Sorel à l'assomption revendiquées qu'on perçoit chez une Martine Sonnet, en passant par la honte exposée par Annie Ernaux ou Didier Éribon.

Dans tous les cas, il m'apparaît que se noue chez les transclasses une défaillance dans l'acquiescientia in se ipso, qui contraste avec l'espèce d'assurance distinguée qui passe pour de la modestie, cultivée chez les membres raffinés des classes dominantes. Les pages qu'Annie Ernaux consacre à l'humiliation éprouvée par cette forme assurée de politesse (cf. parmi d'autres la première page de La Place) provoquent toujours chez moi un ébranlement émotionnel irrépressible qui a pu aller, je le confesse, jusqu'à l'envie, qui est « la haine, en tant qu'elle affecte un homme de telle sorte qu'il est triste du bonheur d'autrui et, au contraire, qu'il est content du malheur d'autrui ${ }^{15}$.

Deuxième exemple: la question éthique qui se pose au transclasse trouve son point d'Archimède, sans doute ici, dans ce défaut quant à l'assurance en soi-même. Point d'Archimède ne signifie pas qu'il en est le vaccin. Le pharmakon sans doute, drogue qui peut guérir ou, sinon tuer, du moins enivrer au point de rendre inconscient du degré de peine que l'on impose aux autres. Mais il y a, dans cet effort vers l'assurance quant à soi, une voie pour une possible reconnaissance rationnelle, ou plutôt pour une reconnaissance de la raison, vers cette générosité dont parle Descartes qui est ce grâce à quoi on s'estime légitimement soi-même. Comme le montre la proposition 52 de la $\mathrm{IV}^{\mathrm{e}}$ Partie de l'Éthique, « la satisfaction de soi-même peut naître de la raison, et seule la satisfaction qui naît de la raison est la plus haute qui puisse exister. » Si je contemple en moi cette puissance de penser qu'est ma raison en actes, alors naît de là cette joie qu'est l'assurance en soimême; et comme cette cause est légitime, cette assurance l'est aussi. Être estimé pour ce que l'on fait ou dit de rationnel ne relève donc pas,

15. Spinoza, Éthique, $\mathrm{III}^{\mathrm{e}}$ partie, définition 23 des affects. 
contrairement à ce que Pascal soutient, de l'illusion tramée par l'orgueil. Rechercher ou recevoir les marques de cette estime ne procède donc pas d'une passivité affective dont il faudrait se guérir, en cherchant à humilier cette activité du moi. Telle serait à mon sens l'impasse d'un Pascal dont l'humilité relèverait du nihilisme chrétien (Nietzsche).

Il s'ensuit que l'ambition peut, à son tour, être une voie pour le parti pris éthique dès lors qu'elle est ambition de raisonner et de faire partager les vérités découvertes par raisonnement. Ainsi, «si quelqu'un cherche trop la gloire, qu'il pense à son usage correct, et à quelle fin il faut la rechercher, et par quel moyen il faut l'acquérir; mais non à son abus, ou à sa vanité ${ }^{16}$. La recherche de la gloire, de l'estime et de l'estime de soi, n'est donc pas condamnable en soi, et peut être légitime éthiquement. À quelle condition ? Si l'ambition est le désir de vouloir conduire tous les autres selon sa propre complexion, ce même désir, guidé par la raison est piétée $e^{17}$. Spinoza nous livre alors une étrange comparaison : l'amant qui imagine posséder la plus belle femme du monde aspire à la fois à ce que tout le monde l'approuve et le craigne en même temps ; au contraire celui qui sait posséder une vérité rationnelle souhaite la partager avec le monde entier : alors, il « agit, non pas par impulsion, mais avec humanité et douceur, et a l'esprit suprêmement en paix ». Nous sommes loin du savant pascalien fier d'avoir résolu une équation, aussi futile que le joueur qui se vante d'avoir mieux placé la balle qu'un autre, pour tromper l'ennui ontologique qui le mine (fragment $136 \mathrm{~L}$ ). Je voudrais simplement remarquer que la plupart des transclasses dont nous parlons, nombre de ceux cités en littérature ou dans les témoignages autobiographiques sont ou ont été des enseignants, ou des animateurs socioculturels. Je ne conclurai pas à leur sagesse, mais seulement qu'ils ont trouvé dans l'institution scolaire le moyen, sans doute de payer la dette -, explication par la moraline des dominants -, mais plutôt de déployer une ambition qui les conduise jusqu'à l'estime de soi et l'assurance en soimême d'abord éprouvées sous le mode de la défaillance. Et c'est sans doute d'avoir fait cette expérience, d'avoir manqué du savoir rationnel comme milieu ambiant, d'avoir manqué de «culture » qu'ils s'efforcent, moins de rembourser une dette ${ }^{18}$, que d'indiquer à des semblables les voies de traverse qui les conduiront mieux qu'eux-mêmes sur les chemins qui mènent à la

16. Éthique, $\mathrm{V}^{\mathrm{e}}$ partie, scolie de la proposition 10.

17. Éthique, $\mathrm{IV}^{\mathrm{e}}$ partie, scolie 1 de la proposition $37 ; \mathrm{V}^{\mathrm{e}}$ partie, scolie de la proposition 5.

18. Je trouve que cette affaire de « remboursement de la dette» de devoir comme dette, sent encore son ton grand seigneur, de ces grands seigneurs qui méprisent le bourgeois ou le commun qui se saigne à rembourser ses dettes, qui méprise le devoir. Dans ce qui peut être ressenti chez les transclasses comme « devoir d'éduquer », plus que la dette il y a, la solidarité avec les semblables, les enfants des milieux populaires. C'est ainsi que Bourdieu, selon un témoignage qui m'a été donné, a préparé, très longtemps, de jeunes étudiants d'origine modeste au concours de l'ENS ou à l'agrégation, en leur donnant des leçons particulières, en leur faisant profiter de son expérience des codes non dits de l'institution, singulièrement de celui qui veut qu'à l'école il faut ne pas être «scolaire », ne pas paraître sans défauts, parce que l'absence de défauts sent trop le labeur, voire le parvenu qui veut masquer ses origines, ce que ne pardonne pas l'institution. 
raison. Et de le faire sans concessions pédagogiques (ou pédagogistes) en refusant d'adapter un enseignement destiné démagogiquement à complaire au désir spontané supposé des classes populaires. C'est là sans doute un point commun avec ces prêtres-ouvriers, figures de transclasse, que j'ai évoqués plus haut.

Toute la difficulté tient à ce que, malgré tout, malgré Spinoza (?), on peut se croire conduit par la raison, en possession d'une vérité démontrée et être animé par la passion d'imposer aux autres sa propre complexion. Il est des enseignants, mêmes transclasses, qui humilient leurs élèves. Mais surtout, hors de l'école, dans le champ politique, il est des transclasses qui, se croyant détenteur d'une vérité rationnelle sont affecté de ce que André Comte-Sponville a nommé le syndrome de l'instituteur : de vrais ambitieux, apprentis tyrans ou vrais dictateurs qui ne peuvent admettre que leurs semblables persévèrent dans ce qu'ils pensent être l'erreur, qui considèrent le peuple comme une masse infantile ignorante qu'ils ont charge d'instruire par tous les moyens. Nous en avons tous rencontré, voire nous en avons été, de ces militants terrifiants de certitude exhibant leur origine modeste en bandoulière en guise d'argument raisonnable, comme d'autres exhibent des quartiers de noblesse : ouvriérisme ou populisme qui ne sont que les figures inversées de la honte sociale dans laquelle ils continuent d'être enfermés.

Pour ne pas conclure, Pascal, encore, pour se souvenir que la conversion de l'ambition en piété est réversible, que la piété peut masquer l'ambition ; ou Molière avec Tartuffe. Ou Desanti pour comprendre comment la capture par la croyance est un effet possible de l'épreuve du manque, comprendre aussi comment le destin philosophique est effort pour se libérer de cette capture. Les dernières pages du livre de Chantal Jaquet, consacrées à l'écriture d'Annie Ernaux ou au style de travail de Bourdieu, achèvent l'enquête dans ce sens, envoyant un rai de lumière rationnelle. 